\title{
An Efficient Method for Eye Detection in Color Images using Morphological Operations
}

\author{
Md. Golam Moazzam \\ Department of CSE \\ Jahangirnagar \\ University, Savar, \\ Dhaka, Bangladesh
}

\author{
Kanija Muntarina \\ Department of CSE \\ Dhaka City College, \\ Dhaka, Bangladesh
}

\author{
Naznin Sultana \\ Department of CSE \\ City University, \\ Bangladesh
}

\author{
Md. Ariful Islam \\ Rana \\ Department of CSE \\ Jahangirnagar \\ University, Savar, \\ Dhaka, Bangladesh
}

\begin{abstract}
This paper presents an efficient method for detecting human eye in a full frontal face color image. Eye regions are characterized by low illumination, high density edges and high contrast as compared to other parts in frontal face image. This method uses basic color image processing and morphological operation to detect eyes. Using color based training algorithm, this technique first extracts skin region of the input image. Then morphological operation is applied for detection of eye boundary and light source reflection of eye, also known as eye dot. It gives finite number of eye candidates from which noise is subsequently removed. For full frontal face images, this method is found highly efficient and accurate for eye detection.
\end{abstract}

\section{Keywords}

Image conversion, Skin detection, Morphological operation, Noise elimination, Eye detection.

\section{INTRODUCTION}

Human eye detection plays an important role in many applications such as iris recognition, eye-gaze tracking, autostereoscopic displays, face detection and recognition. Eyes are the salient feature of the human face. Eye detection becomes important now-a-days in the field of computer vision. Eyes are also important to face expression analysis in the case of human computer interaction. For biometric security based system, iris is the most reliable biometric measure, but it isn't used frequently for the system complexity. For all cases, eye detection is obvious solution for its simplicity compared to iris recognition.

\section{PREVIOUS WORKS}

The eye detection problem has been faced up with various approaches over the last few decades. The commonly used approaches for passive eye detection include the template matching method [1], [2], eigenspace method [3], [4], [5], and Hough transform-based method [6].

In the template matching method, segments of an input image are compared to previously stored images, to evaluate the similarity of the counterpart using correlation values. The problem with simple template matching is that it cannot deal with eye variations in scale, expression, rotation and illumination. Use of multiscale templates was somewhat helpful in solving the previous problem in template matching. A method of using deformable templates is proposed by Yuille et al [7]. This provides the advantage of finding some extra features of an eye like its shape and size at the same time. But the rate of success of this approach depends on initial position of the template. Pentland et al. [5] proposed an eigenspace method for eye and face detection. If the training database is variable with respect to appearance, orientation, and illumination, then this method provides better performance than simple template matching. But the performance of this method is closely related to the training set used and this method also requires normalized sets of training and test images with respect to size and orientation.

Another popular eye detection method is obtained by using the Hough transform. This method is based on the shape feature of an iris and is often used for binary valley or edge maps [6], [8]. The drawback of this approach is that the performance depends on threshold values used for binary conversion of the valleys.

Apart from these three classical approaches, recently many other image-based eye detection techniques have been reported. Feng and Yuen [9] used intensity, the direction of the line joining the centers of the eyes, the response of convolving an eye variance filter with the face image, and the variance projection function (VPF) technique to detect eyes [10]. Zhou and Geng [11] extended the idea of VPF to the generalized projection function (GPF) and showed with experimental results that the hybrid projection function (HPF), a special case of GPF, is better than VPF and integral projection function (IPF) for eye detection. They located the iris using intensity and edge information. They used a feature template, a separability filter, the Hough transform, and template matching in their algorithm. Sirohey and Rosenfeld [12] proposed an eye detection algorithm based on linear and nonlinear filters. Huang and Wechsler's method [13] used genetic algorithms and built decision trees to detect eyes. For the purpose of face detection, Wu and Zhou [14] employed size and intensity information to find eye-analog segments from a gray scale image, and exploited the special geometrical relationship to filter out the possible eye-analog pairs. Using color information to detect eye and it would be useful that give faster image processing.

Although much effort has been spent and some progress has been made, the problem of automatic eye detection is still far from being fully solved owing to its complexity. Factors including facial expression, face rotation in plane and depth, occlusion and lighting conditions, all undoubtedly affect the performance of eye detection algorithms. The method proposed in this paper involves skin detection to eliminate background components followed by eye detection. 


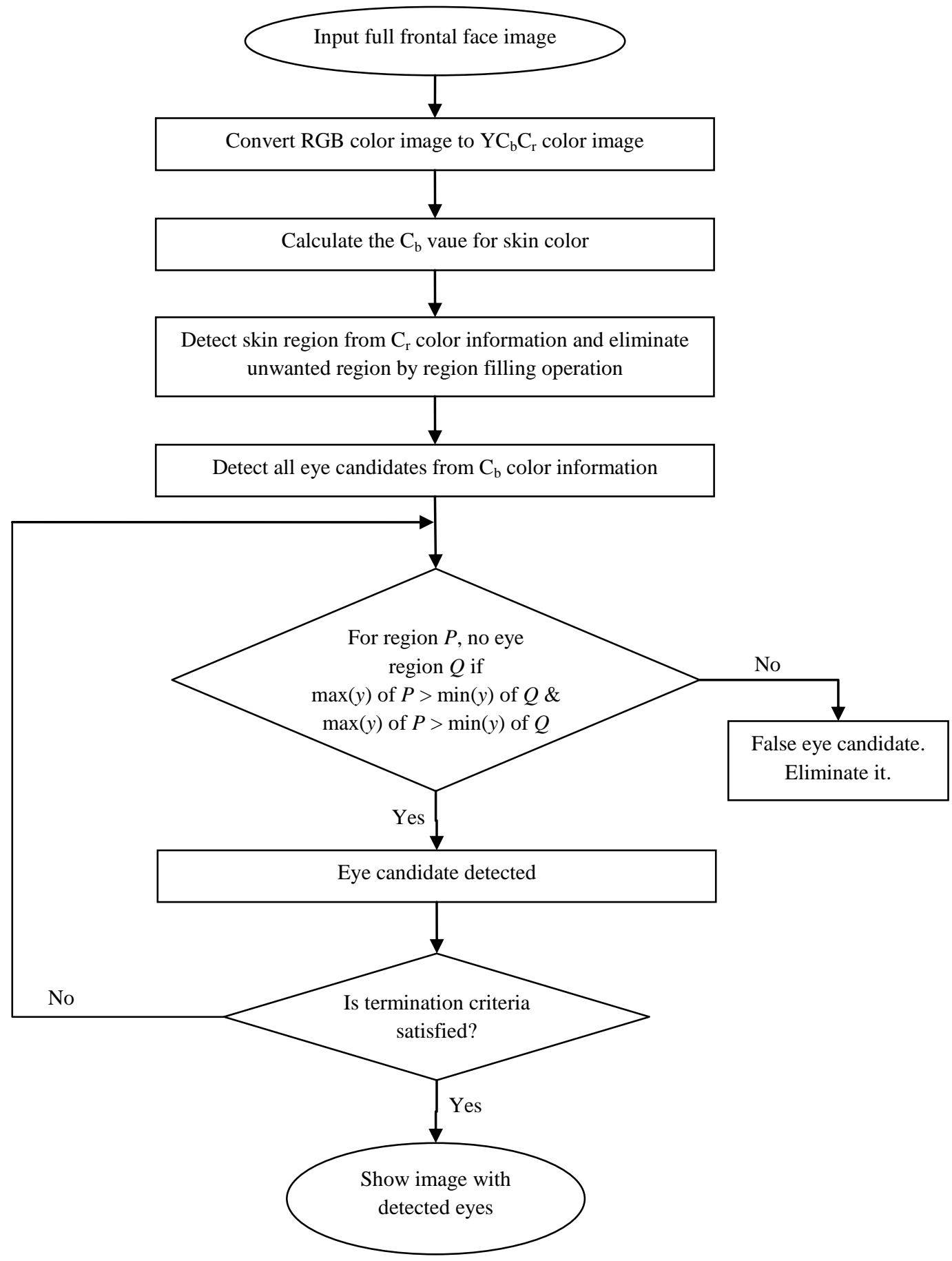

Fig. 1: Flowchart of eye detection method

\section{EYE DETECTION METHODOLOGY}

Eye detection is concerned with determining which part of an image contains the eyes. This requires both high- and lowlevel visual and geometric information processing. The main steps employed for eye detection process are as follows:

Step 1: Input a full frontal face color image with clearly visible a pair of eyes.
Step 2: Convert RGB color image to $\mathrm{YC}_{\mathrm{b}} \mathrm{C}_{\mathrm{r}}$ color space using the following equation:

$$
\left[\begin{array}{lll}
Y & C b & C r
\end{array}\right]=\left[\begin{array}{lll}
R & G & B
\end{array}\right]\left[\begin{array}{ccc}
0.299 & -0.168935 & 0.499813 \\
0.587 & -0.331665 & -0.418531 \\
0.114 & 0.50059 & -0.081282
\end{array}\right]
$$


Step 3: Extracting the skin region by using the color information of $\mathrm{C}_{\mathrm{r}}$. By region filling morphological operation, it can fill any holes on the skin region.

Step 4: Determine all possible eye candidates by $\mathrm{C}_{\mathrm{b}}$ color information.

Step 5: Eliminate all false eye regions by setting a condition and determine the region of eyes on the face.

Flowchart of the proposed eye detection method is shown in Fig. 1.

\subsection{Skin Detection}

Skin region detection is very important in eye detection method. Skin detection optimizes approximate eye position and reduces false eye candidates. To increase the efficiency of eye detection it uses a combination of $\mathrm{RGB}, \mathrm{YC}_{\mathrm{b}} \mathrm{C}_{\mathrm{r}}$, Grayscale and binary image spaces. At first, it needs to manually train to determine the value of skin color. The value of $\mathrm{C}_{\mathrm{r}}$ component used to extract skin region as $\mathrm{YC}_{\mathrm{b}} \mathrm{C}_{\mathrm{r}}$ space is the most efficient space for skin detection. At the time of training, special care must be taken to avoid unwanted value like facial hair and creases. A range of values for skin region extraction is chosen. After extraction, it removes some unnecessary region such as eyebrow region using region filling morphological operation. At last, the whole skin region is obtained as shown in Fig. 2 and Fig. 3.

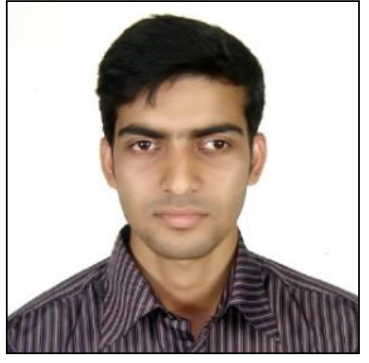

Fig. 2: Original image

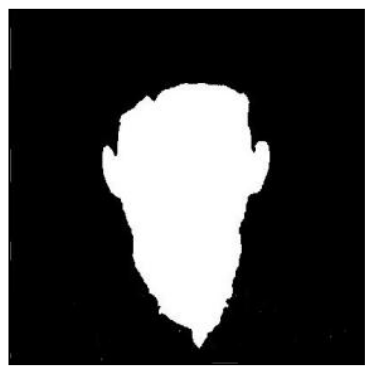

Fig. 3: Skin detection

\subsection{Eye Detection}

An eye has a sharp point of reflection that would be referred to as eye dot or light dot. This point would be clearly visible if eye is properly illuminated. The eye detection technique used here is based on this fact. The $\mathrm{YC}_{\mathrm{b}} \mathrm{C}_{\mathrm{r}}$ format of an image is most efficient in exploiting this light dot. Hence, the input image is first converted into an $\mathrm{YC}_{\mathrm{b}} \mathrm{C}_{\mathrm{r}}$ image and the chrominance values of its pixels are used for further processing.

Next step in eye detection involves eye boundary detection. For this it uses morphological techniques to detect edges. Dilation and then erosion and the calculation of differences between these two methods produces an image with boundaries. The structuring element used in dilation and erosion has a large matrix (9x9 ones) with octagonal shape, so that clear and thick boundaries are detected. This technique is found to be more efficient by using the Canny edge detector. This is followed by suitable thresholding of the image. When the image is thresholded, the light dot is one of the first few candidates that stand out. As thresholding value varies as the image changes, so proper value selection is important, and it is a complex process. To overcome this complexity, an adaptive thresholding technique is used. This value is calculated by an iterative process. Starting from the value of 220 on a 255 scale and is decreased by 5 per iteration. The aim of the iteration process is to obtain a minimum number of four and a maximum number of six blobs. It was observed that thresholding is the most probable to output both eyes and with minimum noise. Morphological closing operation is done after thresholding. It improves the ease of eye detection and eliminates stray points. Some of the blobs obtained from the image are too small, and some are too large. Both are not the candidates of an eye and that is why these should be morphologically eliminated. This is followed by eliminating long and slender blobs, either horizontal or vertical ones, as these blobs are certainly not eyes. Morphological binary image processing is used again. Using an 'And' operation, this image and skin image are combined. As eyes invariably lie in the face region, so only the common candidates survive. This whole process is included in a loop and at the end of the loop the number of emerging candidates is checked. The threshold value is changed for the next loop, if this number is not between four and six.

Now, the job is to find the two blobs or regions that represent the eye region and with this remove the noise or false eye candidates. As the input image is the full frontal face image, so eyes lie more or less in the same horizontal line.

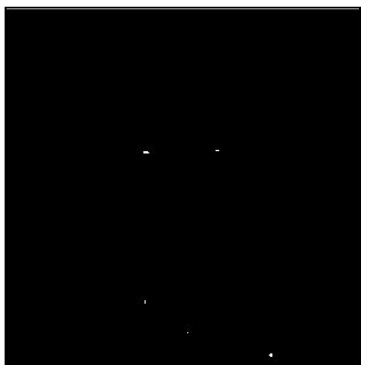

Fig. 4: Eye candidates

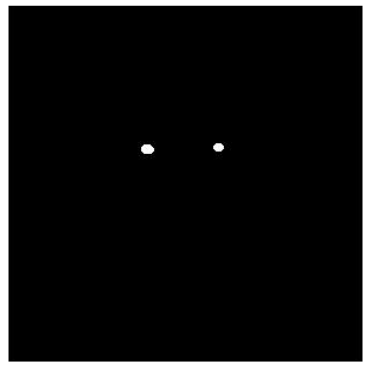

Fig. 5: Detected eye region

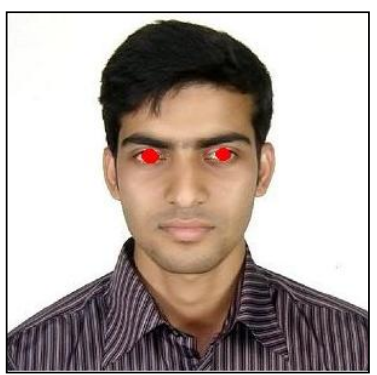

Fig. 6: Highlighted eye location

Hence, the condition is imposed that for a region $P$, if there is no region $Q$, such that the maximum $y$ value of $P$ is greater than the minimum $y$ value of $Q$, and the maximum $y$ value of $Q$ is greater than the minimum $y$ value of $P$, then $P$ is not a candidate for eye. Another condition is imposed on the lower limit and upper limit of the distance between $P$ and $Q$. This eliminates most of the noise points. Now, the main problem arises if there are three points in a line. Many times, it is observed that two of the three points are very close to each other, and actually both are part of the same eye. This problem is solved by horizontal dilation, which connects the two points and finally determines the two candidates, which are eyes. The process is depicted in Fig. 4, Fig. 5 and Fig. 6.

\section{EXPERIMENTAL RESULTS}

The effectiveness of this approach has been justified using images of different sizes and quality. When an image is subjected in the input, it highlights the eye location of the 
image. Some experimental results are shown in Fig. 7 and Fig.

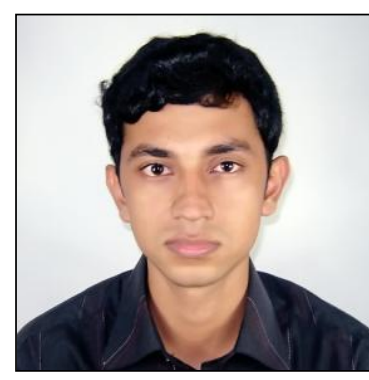

(a)

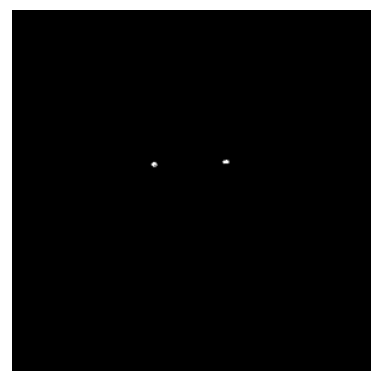

(c)

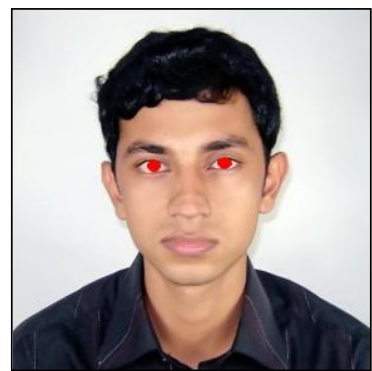

(e)

Fig. 7: (a) Input Image (b) Skin detection (c) Eye candidates (d) Detected eye region (e) Highlighted eye location

\section{PERFORMANCE ANALYSIS}

The proposed eye detection algorithm involves color image processing and morphological operation. The technique is found to be highly efficient and accurate for detecting eyes in frontal face images. The algorithm has been examined with images of different sizes and quality. Almost in all cases, experiments have shown very promising results. Table I summarizes the performance analysis of this method.

Table I. Performance measurement for different size and quality of images.

\begin{tabular}{|c|c|c|c|}
\hline $\begin{array}{c}\text { Input Image } \\
\text { Dimension } \\
\text { (pixel) }\end{array}$ & $\begin{array}{c}\text { Process } \\
\text { Time (s) }\end{array}$ & $\begin{array}{c}\text { Eye } \\
\text { Detected? }\end{array}$ & Accuracy \\
\hline 300 X 300 & 3.8125 & Yes & $100 \%$ \\
\hline 600 X 600 & 9.8125 & Yes & $100 \%$ \\
\hline 318 X 398 & 3.9219 & Yes & $100 \%$ \\
\hline $224 \times 224$ & 2.1094 & No & - \\
\hline $198 \times 198$ & 1.7969 & Yes & $100 \%$ \\
\hline
\end{tabular}

The measurement is done in MATLAB 7.8 and with Core i3 processor. Here, this performance measurement is categorized by two ways. Process time depends on image dimension and detection accuracy depends on image quality.

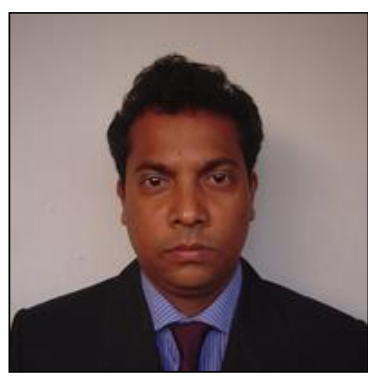

(a)

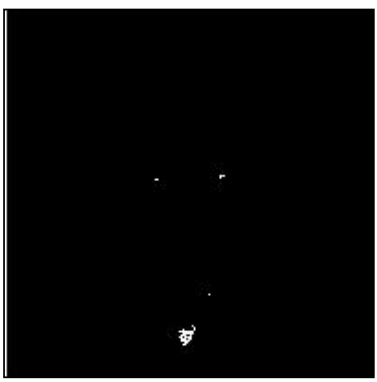

(c)

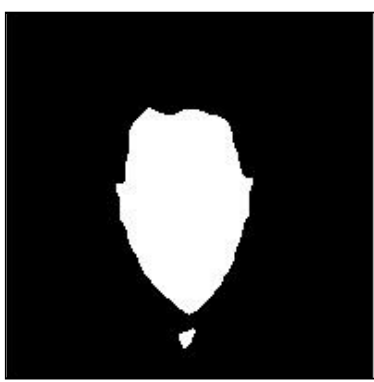

(b)

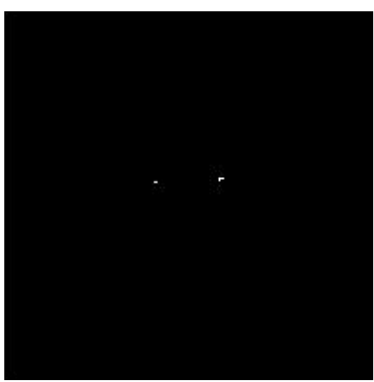

(d)

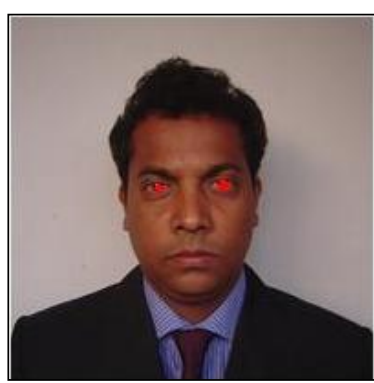

(e)

Fig. 8: (a) Input Image (b) Skin detection (c) Eye candidates (d) Detected eye region (e) Highlighted eye location

\section{CONCLUSION AND FUTURE WORK}

In this work a simple and efficient eye detection method is implemented. It is based on robust skin detection and followed by morphological operation to detect eye. It is observed from the results that this technique is successful for $90 \%$ of frontal face images, which show two clearly illuminated eyes. Future work can be extended as eye gaze detection and tracking and image can be inputted by a webcam. Further improvement also includes detection of eyes in multiple faces, and faces with different orientations.

\section{REFERENCES}

[1] R. Brunelli, T. Poggio, "Face Recognition, Features versus templates", IEEE Trans. Patt. Anal. Mach. Intell. 15(10)(1993) 1042-1052.

[2] D. J. Beymer, "Face Recognition under varying pose", IEEE Proceedings of Int. Conference on Computer Vision and Pattern Recognition (CVPR'94), Seattle, Washington, USA,1994, pp.756-761. 
[3] Jiatao Song, Zheru Chi, and Jilin Liu, "A Robust Eye Detection Method Using Combined Binary Edge and Intensity Information", Science Direct, Pattern Recognition 39 (2006) 1110-1125.

[4] R. C. Gonzalez and R. E. Woods, "Digital Image Processing", 2nd edition, PHI

[5] A. Pentland, B. Mognanddam, T. Starner, "View based and modular eigenspaces for face recognition", IEEE Proceedings of Int. Conference on Computer Vision and Pattern Recognition (CVPR'94), Seattle, Washington, USA, 1994, pp.756-761.

[6] K. M. Lam, H. Yan, Locating and extracting eye in human face images, Pattern Recognition, 29(5)(1996) 771-779.

[7] A. L. Yuille, P. W. Hallinan, D. S. Cohen, "Feature Extraction From Faces Using Deformable Template", Int. J. Comput.Vision 8(2)(1992) 99-111.

[8] G. Chow, X. Li, "Towards a system of automatic facial feature detection", Pattern Recognition (26)(1993) 1739-1755.
[9] G. C. Fang, P. C. Yuen, "Multi cues eye detection on gray intensity image", Pattern Recognition (34)(2001) 1033-1046.

[10] G. C. Fang, P. C. Yuen, "Variance Projection function and its application to detection for human face recognition", Pattern Recognition Lett. 19(1998) 899906.

[11] Z. H. Zhou, X. Geng, "Projection functions for eye detection", Pattern Recognition (37)(5)(2004) 10491056.

[12] S. A. Sirohey, A. Rosenfield, "Eye detection in a face image using linear and non linear filters", Pattern Recognition (34)(2001)(1367-1391)

[13] J. Huang, H. Wechsler, "Visual routines for eye localization using learning and evolution", IEEE Trans. Evol. Comput. (4)(1)(2000) 73-82.

[14] J. Wu, Z. H. Zhou, "Efficient face candidate selector for face detection", Pattern Recognition(36)(2003) 1175-1186. 